\title{
Optimal Interpolation of Satellite and Ground Data for Irradiance Nowcasting at City Scales
}

\author{
Antonio T. Lorenzo ${ }^{\mathrm{a}, *}$, Matthias Morzfeld $^{\mathrm{b}}$, William F. Holmgren ${ }^{\mathrm{c}}$, Alexander D. Cronin ${ }^{\mathrm{d}, \mathrm{a}}$ \\ ${ }^{a}$ University of Arizona, College of Optical Sciences, 1630 E. University Blvd., Tucson, AZ 85721, United States \\ ${ }^{b}$ University of Arizona, Department of Mathematics, 617 N. Santa Rita Ave., Tucson, AZ 85721, United States \\ ${ }^{c}$ University of Arizona, Department of Hydrology 83 Atmospheric Sciences, 1118 E. 4th Street, Tucson, AZ 85721, \\ United States \\ ${ }^{d}$ University of Arizona, Department of Physics, 1118 E. 4th Street, Tucson, AZ 85721, United States
}

\begin{abstract}
We use a Bayesian method, optimal interpolation, to improve satellite derived irradiance estimates at cityscales using ground sensor data. Optimal interpolation requires error covariances in the satellite estimates and ground data, which define how information from the sensor locations is distributed across a large area. We describe three methods to choose such covariances, including a covariance parameterization that depends on the relative cloudiness between locations. Results are computed with ground data from 22 sensors over a $75 \times 80 \mathrm{~km}$ area centered on Tucson, AZ, using two satellite derived irradiance models. The improvements in standard error metrics for both satellite models indicate that our approach is applicable to additional satellite derived irradiance models. We also show that optimal interpolation can nearly eliminate mean bias error and improve the root mean squared error by $50 \%$.
\end{abstract}

Keywords: Solar irradiance, optimal interpolation, data assimilation, nowcasting

\section{Introduction}

Estimates of global horizontal irradiance (GHI) are essential at many stages of photovoltaic (PV) system deployment and operation. A widely used 5 technique is to compute GHI from geostationary satellite images, which are typically available every 15 to 30 minutes and cover large areas of the globe. Such satellite derived estimates of GHI are commonly used to design and site PV power plants $(\overline{\mathrm{Vi}}-$ 10 gnola et al. 2013), to forecast the output of a fleet of PV generators (Kühnert et al., 2013), and to provide real-time estimates of distributed generation (DG) or "behind the meter" generation of rooftop PV systems (Saint-Drenan et al., 2011). Satellite derived estimates have also been used to detect failures in PV systems (Drews et al. 2007 ).

In addition to satellite derived GHI estimates, one may have access to ground sensors that provide more accurate GHI measurements, but are of-

\footnotetext{
* Corresponding author

Email address: atlorenzo@email.arizona.edu (Antonio T. Lorenzo)

Preprint submitted to Solar Energy
}

20 ten sparsely distributed. We present a method that combines the broad areal coverage of satellite derived GHI with the accurate point measurements from ground sensors in order to provide more accurate GHI estimates for city-scale areas.

Similar techniques have used ground measurements to improve satellite derived irradiance estimates in the context of improving daily (or longer) irradiance estimates. Much of this work studies so called site adaptation techniques with the goal of improving multi-year satellite irradiance estimates using a limited measurement campaign from ground sensors (Polo et al. 2016). A number of studies use Kriging methods that rely on spatial interpolation of the ground data along with satellite derived estimates (D'Agostino and Zelenka, 1992 Journée et al., 2012; Frei et al.||2015). Others use linear bias corrections (Polo et al., 2015), polynomial bias corrections (Mieslinger et al., 2014), or apply a polynomial to correct the satellite cumula40 tive distribution function (Schumann et al., 2011). Ruiz-Arias et al. (2015) used optimal interpolation (OI) with numerical weather prediction solar radi-

December 27, 2016 
ation data and monthly-averaged daily GHI values from ground sensors.

OI is a Bayesian technique often used in geophysics, in particular numerical weather prediction, to combine models and observations. OI is mathematically equivalent to $3 \mathrm{D}$ variational methods, kriging, and Gaussian process regression (Low et al. 2015). OI and 3D variational techniques are often used in the field of meteorology, kriging is used in the context of geostatistics, and one often encounters Gaussian process regression in the context of machine learning. Thus, each method seeks 55 a solution with the approach and quantities, like covariances, appropriate for each context.

In the context of this study, the satellite derived GHI estimates represent the model and the ground sensor data are the observations for OI. We 60 focus on improvements to GHI estimates from a single satellite image using OI rather than improving the multi-year satellite estimates. This single satellite image will be used to nowcast DG production and to produce forecasts. We also present a 65 novel method to parameterize the correlation between satellite pixels using the relative cloudiness between them.

We describe the satellite derived irradiance models and observation data in Section 2, the OI 70 method in Section 3 , and three ways to estimate covariances between locations in Section 4. These covariances are critical to the success or failure of OI. A method to correct for satellite geolocation errors is described in Section 5 , and parameter tun-

75 ing is detailed in Section 6. We present and discuss the results of applying OI to Tucson, AZ in Section 7. Finally, a summary of the work is provided in Section 8

\section{Models and Observations}

\section{2.1. Satellite Derived Irradiance Models}

To investigate how well OI works with differ- ${ }^{110}$ ent types of prior information, we use two different models to convert satellite images to GHI maps. The resulting GHI maps are called the 85 "background" or "prior" in OI and will be denoted by $\mathbf{x}_{b}$. Both models use images from the GOES-W geostationary satellite that cover the city of Tucson, AZ (roughly $75 \times 80 \mathrm{~km}$ ). An example of a visible albedo image derived from the visible channel of GOES-W is shown in Fig. 1 .

One of the models is a physical model called 120 the University of Arizona Solar Irradiance Based

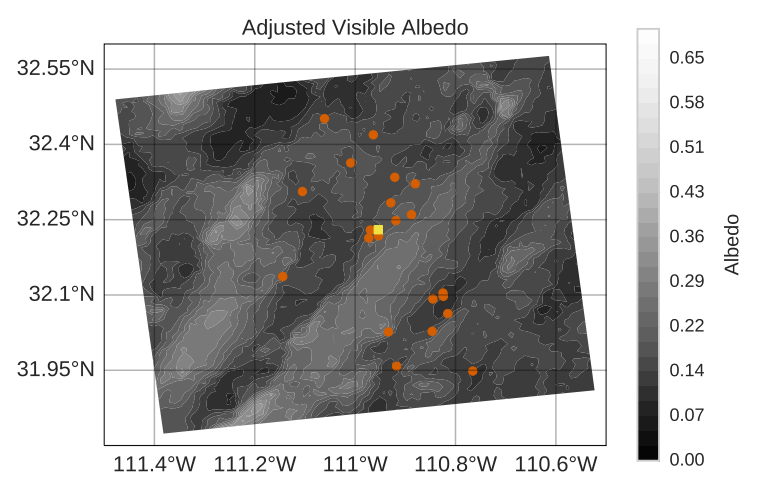

Figure 1: Visible albedo image derived from the visible channel of the GOES-W satellite. Lighter colors indicate cloudier areas. The orange dots represent the locations of the sensors used in this study which includes both irradiance sensors and rooftop PV systems as described in Section 2.2. The yellow square in the center indicates the location of a calibrated GHI sensor on the University of Arizona campus. The image covers an area of roughly $75 \times 80 \mathrm{~km}$ over Tucson, AZ

on Satellite (UASIBS) model (Kim et al., 2016). UASIBS uses the visible and infrared images from 95 the GOES-W satellite to generate a cloud mask. Then, parameterized cloud properties determined from the infrared images are used in a radiative transfer model to determine the surface GHI. This GHI estimate has the same resolution as the visible channel of the GOES-W satellite (approximately 1 $\mathrm{km})$.

The second model is a semi-empirical model, which we refer to as the SE model. This model is based on the SUNY model which applies a regres05 sion to the visible channel of the GOES-W satellite (Perez et al. 2002). The only differences between our SE model and the SUNY model are that the dynamic range is set with the 3 months of data used in this study instead of the recommended 60 day window with seasonal corrections and that the specular correction factor was neglected.

To remove effects of the diurnal cycle and ease incorporation of data from rooftop PV systems, all images were converted into clear-sky index images by dividing the estimated GHI by a clear-sky GHI estimate. The resulting values of clear-sky index range from nearly 0 for an overcast sky to 1 for a cloud-free sky. These conversion algorithms do not take into account image time-stamp inaccuracies or satellite geolocation errors, but corrections for those errors will be discussed in Section 5 . 


\subsection{Ground Observations}

The observation data are collected from 22 sensors including a calibrated NREL MIDC sensor

125 (Wilcox and Andreas, 2010), custom irradiance sensors (Lorenzo et al. 2014), and data from rooftop PV systems. The sensor locations are indicated by orange circles in Fig. 1

Irradiance observations were averaged to 5 min-

130 utes to match $\mathrm{PV}$ data that are reported as 5 minute averages. This averaging is consistent with the inherent averaging due to the satellite spatial resolution. We note that all data sources (ground sensors and satellite images) are available in near

135 real-time so that the OI corrected GHI images can be used as a basis for forecasts or DG nowcasts.

All data were converted to clear-sky index data using clear-sky expectations for each sensor. To produce the clear-sky expectation for one day, the 140 measurements from preceding clear days within one week are averaged to produce an initial estimate. This initial estimate is then scaled to match the 190 clear times on the day of interest to account for differences in turbidity or temperature. This method 145 simplifies the calculation of clear-sky expectations for the rooftop PV systems because no parameters about the system (directional response, peak power) are assumed. The clear-sky expectations and clear-sky index data was inspected manually 150 to confirm the quality. Note that the ground observation data may experience cloud enhancement events which lead to clear-sky indices greater than 1.

We restrict our data and analysis to solar zenith 155 angles less than 60 degrees. At times, we also withhold sensors from the OI routine and use these sensors to validate how well OI performs for other locations in the image besides the input sensor locations.

\subsection{Data Set Description}

About 1300 satellite images collected over April, May, and June 2014 were converted to irradiance images with the two models and paired with the 205 corresponding ground observations. We randomly divide the data set into a training set with 437 images (252 clear and 185 cloudy images) and a verification set of 874 images (504 clear and 370 cloudy images). The training set is used to tune param- 210 eters for OI as described in Section 6. The verification set is used for error analysis and to draw conclusions about OI.
The distinction between clear and cloudy satellite images will become important in Section 3 for determining sensor error variances. Clear times are identified using a combination of the UASIBS estimates and the ground sensor data. Specifically, if the minimum value of a UASIBS clear-sky index image is greater than 0.8 , the mean of the image is greater than 0.99, and the second largest deviation from 1 of any of the ground observations is less than 0.05 , then we classify the image as clear. This procedure accurately identifies times at which no clouds exist in the area of study. Other methods can also be used to perform this classification Reno and Hansen, 2016, Escrig et al., 2013: Ghonima et al.| 2012), but our simple method is sufficient for our purposes.

\section{Optimal Interpolation}

We now describe the OI method. Under wide assumptions, OI is optimal in the sense that it is the best linear, unbiased estimator of a field. Further detail can be found in data assimilation textbooks, e.g. Kalnay (2003).

The result of the OI routine, known as the analysis, $\mathbf{x}_{a}$, is a vector that is produced by computing a weighted sum of the background (or prior information), $\mathbf{x}_{b}$, and a correction vector (or "innovation" in $\mathrm{OI}$ ) that depends on the measurements, $\mathbf{y}$ :

$$
\mathbf{x}_{a}=\mathbf{x}_{b}+\mathbf{W}\left(\mathbf{y}-\mathbf{H} \mathbf{x}_{b}\right)
$$

As discussed in Section 2.1 the $N$ satellite derived clear-sky indices from one image are represented as the background vector, $\mathbf{x}_{b}$. The measurement vector, $\mathbf{y}$, is a vector of length $M$ of clear-sky indices generated from $M$ ground irradiance sensor and rooftop PV power data observations as discussed in Section 2.2. The observation matrix, $\mathbf{H}$, is an $M \times N$ matrix that maps points in the background space to points in the observation space. We construct $\mathbf{H}$ using the nearest neighbor approach of selecting the satellite pixels that are closest to the observation locations. Another possible approach is to average the points in the background that are within a given radius of each sensor location. Furthermore, $\mathbf{H}$ can contain conversion factors to convert the units of $\mathbf{x}_{b}$ to the units of $\mathbf{y}$. In our case however, $\mathbf{H}$ is unitless because $\mathbf{y}$ and $\mathbf{x}_{b}$ are both in units of clear-sky index. Example background and analysis images for the UASIBS and SE models are shown in Fig. 2 . 

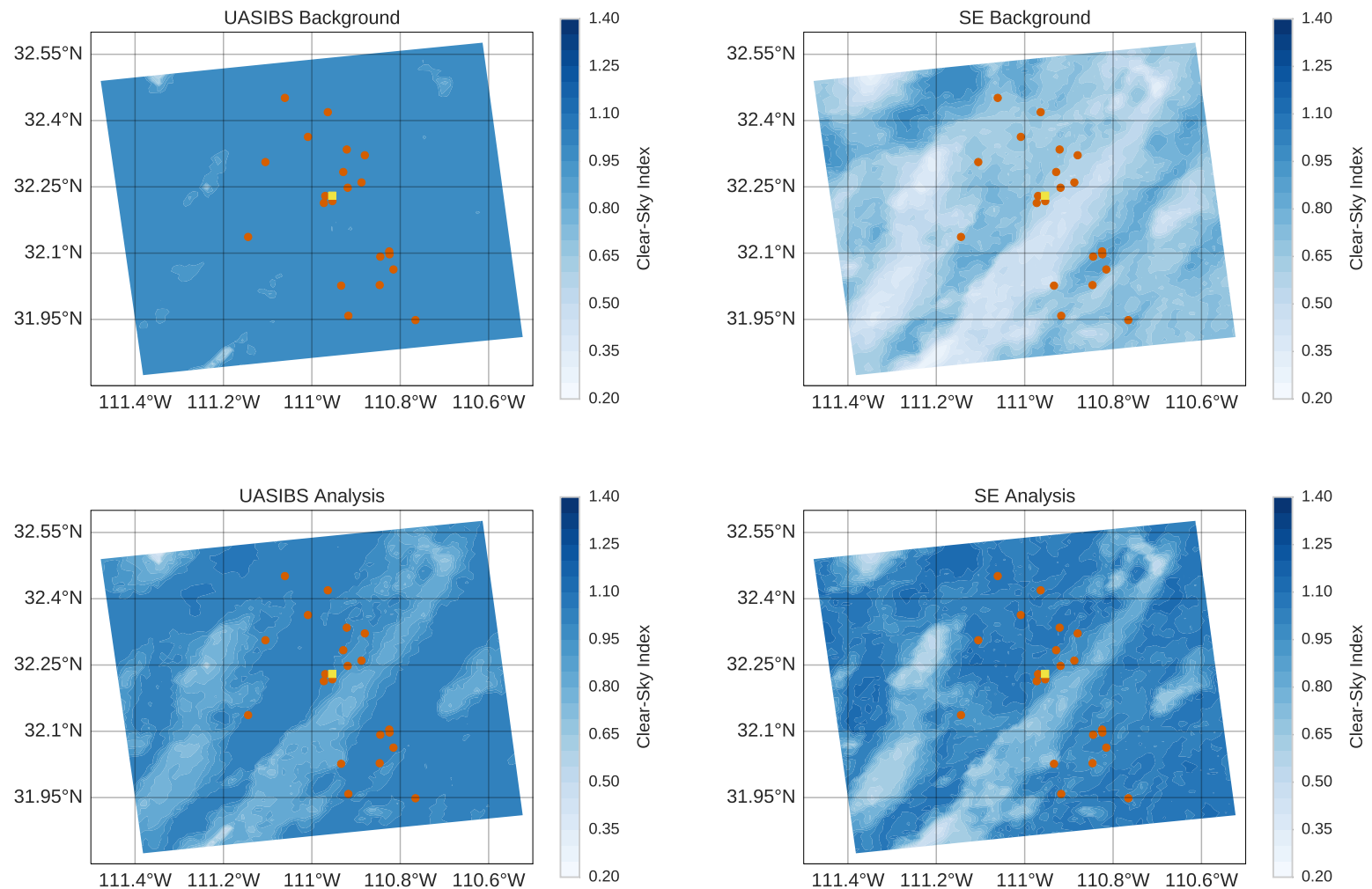

Figure 2: Example background (top row) and analysis (bottom row) clear-sky index images using the UASIBS (left column) and SE (right column) satellite image to ground irradiance models applied to the visible satellite image shown in Fig. 1 Note that in this case, UASIBS failed to produce many clouds. OI adds clouds to the analysis and also makes the darker, clear areas even more clear. In this case, the SE model overproduces clouds. OI reduces the cloud amount while keeping clouds in suitable locations.

The weight matrix, $\mathbf{W}$, is an $N \times M$ matrix constructed from the error covariance matrices of the background, $\mathbf{P}$, and the observations, $\mathbf{R}$, as

$$
\mathbf{W}=\mathbf{P H}^{T}\left(\mathbf{R}+\mathbf{H} \mathbf{P} \mathbf{H}^{T}\right)^{-1} .
$$

Choosing these error covariance matrices must be done with care: they define how information is transferred from sensor locations to other locations in the satellite image, and how much weight is given to any one sensor or satellite pixel.

$\mathbf{R}$ is defined as the error covariance matrix of the observations such that

$$
\mathbf{y}=\mathbf{y}_{t}+\mathbf{e}, \quad \mathbf{e} \sim N(\mathbf{0}, \mathbf{R})
$$

where $\mathbf{y}_{t}$ is the true value of the observation and
On clear days, we assume the true clear-sky index values are 1.0. We also assume that the measurements are unbiased and that the correlations in the agonal matrix in our case. Thus, we estimate the diagonal elements (sensor error variances) by computing the variance on a set of clear days in the training data set for each sensor individually. Furthermore, we restrict the minimum variance to be 0.001 or about a $3 \%$ clear-sky index RMS error to avoid exact interpolation at sensor locations. With $\mathbf{R}$ calculated from the ground sensor data, we describe various ways to parameterize $\mathbf{P}$ next. 


\section{Covariance Parameterization and Corre- lation Structure}

Choosing an appropriate background error covariance matrix is an important step in the OI method for this application and determines how well OI performs. The background error covariance matrix, $\mathbf{P}$, defines how information is transferred from sensor observation locations to locations throughout the background image. Similar to $\mathbf{R}, \mathbf{P}$ is defined such that

$$
\mathbf{x}_{b}=\mathbf{x}_{t}+\mathbf{g}, \quad \mathbf{g} \sim N(\mathbf{0}, \mathbf{P})
$$

where $\mathbf{x}_{t}$ is the "true" value of the satellite derived clear-sky index image and $\mathbf{g}$ is a random vector sampled from a multivariate normal distribution with 240 mean 0 and covariance $\mathbf{P}$.

We will now describe three methods to calculate $\mathbf{P}$ :

1. Empirical: $\mathbf{P}$ calculated empirically from all of the background images,

245 2. Spatial: $\mathbf{P}$ with correlations parameterized based on the physical distance between pixels, and

3. Cloudiness: $\mathbf{P}$ with correlations parameterized based on the difference in cloudiness between each pixel.

\subsection{Empirical Covariance}

The empirical $\mathbf{P}$ is calculated by assuming that satellite-derived clear-sky index images are sampled from the same multivariate normal distribution and then simply computing the covariance using all images in the training data set. This assumption is likely invalid given the high probability of clear days leading to a non-Gaussian distribution. An analysis computed with this type of $\mathbf{P}$ gives non-physical 260 results, as described in Section 7, but is included for comparison.

\subsection{Correlation Matrix Parameterization}

Before describing spatial and cloudiness covariances, it is useful to decompose $\mathbf{P}$ into a diagonal variance matrix, $\mathbf{D}$, and a correlation matrix, $\mathbf{C}$ as

$$
\mathbf{P}=\mathbf{D}^{1 / 2} \mathbf{C D}^{1 / 2} .
$$

Here, D sets the scale of the errors while $\mathbf{C}$ describes how errors and information spread. We obtain $\mathbf{D}$ in a similar manner as we do for $\mathbf{R}$, we use a number of clear images from the training data set to estimate the variance of each pixel in the background individually. The errors in $\mathbf{x}_{b}$ come mainly from the satellite image to ground irradiance conversion that often exhibits large differences in error between clear and cloudy images. Thus, we allow for a tunable scaling factor, $d$, in the construction of D for cloudy images to account for possible model error differences between clear and cloudy skies so that

$$
\mathbf{D}=d \mathbf{D}^{\prime}
$$

where $\mathbf{D}^{\prime}$ is the variance estimated from the clear images.

The correlation matrix $\mathbf{C}$ defines how information is transferred from the sensor locations to other locations in the satellite estimate. $\mathbf{C}$ can be parameterized based on the spatial distance between points in the background as in Ruiz-Arias et al. (2015) or, as we demonstrate, one might rely on information in the current satellite image, such as cloudiness.

To construct the elements of $\mathbf{C}, c_{i j}$, we apply a correlation function, $k$, to the distance metric $r$ computed between each pixel $i$ and $j$

$$
c_{i j}=k\left(r_{i j}\right)
$$

Any number of covariance functions, $k$, can be chosen; see Rasmussen and Williams (2005) for a partial list. We chose to study a piece-wise linear correlation function

$$
k(r)=\left\{\begin{array}{ll}
1-\frac{r}{l} & r<l \\
0 & r \geq l
\end{array},\right.
$$

an exponential correlation function

$$
k(r)=\exp \left(-\frac{r}{l}\right)
$$

and a square exponential correlation function

$$
k(r)=\exp \left(-\frac{r^{2}}{l^{2}}\right) .
$$

For each correlation function, $l$ is a characteristic length that we tune with a training data set for each 75 choice of $k$ to minimize error as described later in Section 6 .

\subsection{Spatial Covariance}

The distance metric for the spatial correlation parameterization is the standard Euclidean distance 
(once locations are mapped to a two dimensional plane using a map projection),

$$
r_{i j}=\sqrt{\left(x_{i}-x_{j}\right)^{2}+\left(y_{i}-y_{j}\right)^{2}} .
$$

Thus, the spatial covariance $\mathbf{P}$ is constructed by applying Eqs. (5) to (7) and (11) with a tuned $k, l$, and $d$ as described in Section 6 .

\subsection{Cloudiness Covariance}

For what we call cloudiness covariance, we parameterize $\mathbf{C}$ based on the difference in cloudiness in the visible satellite image. This corresponds to 285 only adjusting the cloudy areas with observations that are experiencing similarly cloudy sky and leaving the clear areas to be adjusted by observations of the clear sky. This adjustment is made without consideration of the spatial distance between pixels. 290 We use the adjusted visible albedo calculated from the visible satellite image rather than the processed clear-sky index maps to compute the correlation. This avoids cloud representation errors that may arise in the satellite to irradiance conversion; for 295 example, note how UASIBS fails to produce clouds in many areas of Fig. 2. Also note that because this parameterization depends on the visible satellite image, $\mathbf{C}$ and subsequently $\mathbf{P}$ are calculated for each image individually.

To calculate the adjusted visible albedo, we convert the visible brightness counts from the satellite, $b_{i}$, to visible albedo and divide by the cosine of the solar zenith angle, $\phi$, to correct for the time of day:

$$
v_{i}=\left(\frac{b_{i}}{255}\right)^{2} / \cos \left(\phi_{i}\right) .
$$

An example of this adjusted visible albedo is shown in Fig. 1. We also remove the constant (over the three months we studied) background albedo that is due to the land surface. This background is calculated as the average of the adjusted visible albedo on clear days in the training set so that

$$
z_{i}=v_{i}-\bar{v}_{i}^{\text {clear }}
$$

The distance metric for the cloudiness correlation parameterization is the absolute value of the difference between pixel values of the adjusted visible albedo image (with the land surface background removed):

$$
r_{i j}=\left|z_{i}-z_{j}\right| \text {. }
$$

Thus, the cloudiness covariance $\mathbf{P}$ is constructed by applying Eqs. (5) to (7) and (12) to (14) with a 350 tuned $k, l$, and $d$ for each individual satellite image.

\subsection{OI Summary}

In summary, to perform OI, one must first collect 305 fine observation error covariances, $\mathbf{R}$, from the observation data and define the background error covariances, $\mathbf{P}$, either empirically or by following the above procedure after choosing a distance metric $r$, the correlation function $k$, the correlation length $l$, and the scaling factor $d$ using Eqs. (5) to (7). Finally, Eqs. (1) and (2) can be used to compute the analysis, $\mathbf{x}_{a}$.

\section{Geolocation Correction}

It is important to consider errors in the tagged location for each satellite pixel compared to the ground sensors and the time-stamp of the image. Furthermore, one must take into account the position of the sun in order to predict the cloud shadow 320 location on the ground. If this cloud shadow location is inaccurate, the optimal interpolation routine may perform poorly, or worse, may invert the cloudy and clear areas of the images. Examples of an inverted analysis and the corrected analysis once these position adjustments are taken into account are shown in Fig. 3 .

The first geolocation issue is called parallax, which is the discrepancy between the actual location and the location tagged by a satellite due to 330 the satellite viewing the scene at an angle (Vicente et al. 2010). The GOES-W satellite is located at $135^{\circ} \mathrm{W}$ on the equator while Tucson, $\mathrm{AZ}$ is at roughly $32^{\circ} \mathrm{N}$ and $110^{\circ} \mathrm{W}$. The satellite tags the location of each pixel as if it were at the surface. This means, for our region, that a cloud obscures a pixel that is to the NE of the cloud. Thus, the actual location of the cloud is to the SW of what the satellite tags the pixel as.

Another source of error is a timing issue that 340 arises because the satellite tags each image with a single time, however it may take the satellite 30 minutes to sweep and capture that image. Thus, there is uncertainty in the time that any part of the image was captured.

Estimating where the cloud shadow falls on the surface due to solar position effects is the final geolocation issue we take into account. If the shape and height of the clouds is known, the correction for both parallax and solar position is a simple geometry problem. However, cloud shape and height are difficult to determine with sufficient accuracy, 

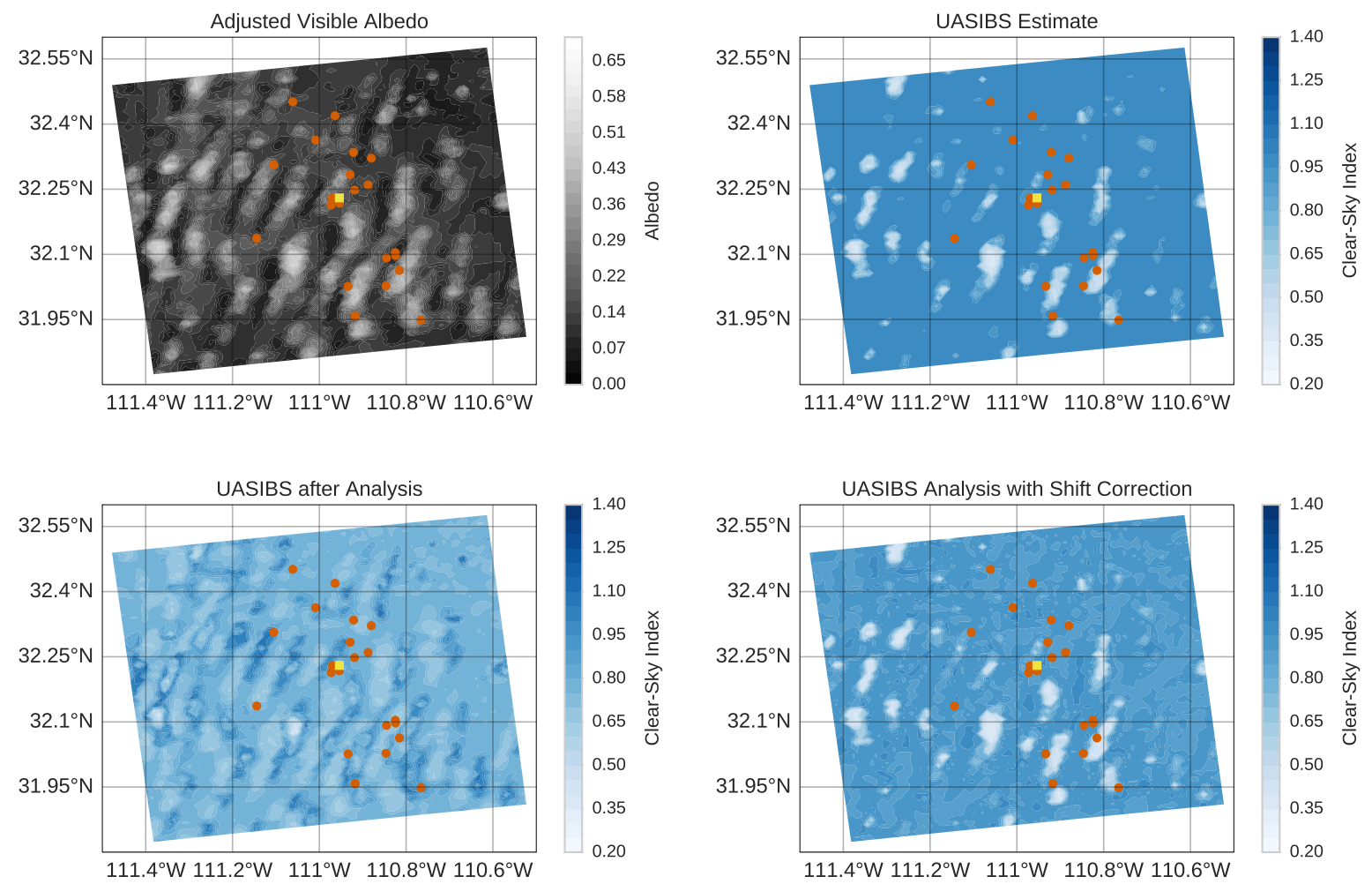

Figure 3: An example of a time when errors in geolocation of the satellite image result in an analysis that is inconsistent with the actual satellite image. The background estimate in this case (upper right) agrees well with the visible satellite image (upper left). However, after performing OI, the analysis (lower left) has clouds in areas that should be clear according to the visible image and sometimes makes areas that should have clouds clear. After shifting the background image slightly, OI produces an analysis (lower right) that is consistent with the visible image. 
and we rely only on an estimate of the height of the 390 top of the clouds and ignore the vertical thickness. We also assume that the cloud height is uniform in 355 one image.

Given these limitations, we use a simple strategy to correct for geolocation errors. We find a sin- 395 gle optimal cloud height by minimizing the mean squared error (MSE) between the OI analysis and 360 sensors that are not used to perform OI. The sensors not used are the same cross-validation sensors we will discuss next. We perform this correction 400 using a grid search for cloud heights ranging from 0 to $14 \mathrm{~km}$ and we shift the entire background image 365 based on that height, perform OI, then calculate the MSE. Once the height that minimizes MSE is found, we perform OI again on the shifted back- 405 ground image and save the analysis as our result for the given time. This technique assumes that there 370 is a single cloud layer, which is not always the case and can be improved in the future.

\section{Tuning OI to a Specific Location}

As discussed in Section 4.2, $k, l$, and $d$ are tunable parameters that determine how information is spread through the image. In order to find suit- ${ }^{41}$ able values of these parameters, we split the satellite images into a training and a verification set as described in Section 2.3, and tuning is only performed with the training set. Furthermore, we perform a six fold cross-validation over the sensors in order ${ }^{42}$ to validate the model at locations not included in the OI calculation. We then perform a grid search through the parameter space and define the optimal parameters as those that give the lowest mean (over the cross-validation sets) MSE of the withheld ${ }^{425}$ sensors. This tuning is performed for both spatial and cloudiness correlation parameterizations and for both the SE and UASIBS models.

Table 1: Optimal parameters for the UASIBS and SE models for both cloudiness and spatial covariances. $l$ has units of adjusted visible albedo for cloudiness covariances and units of kilometers for spatial covariances.

\begin{tabular}{lllll}
\hline & & $d$ & $l$ & $k$ \\
\hline \multirow{2}{*}{ UASIBS } & Cloudiness & 156 & 0.2 & linear \\
& Spatial & 225 & 20 & exp. \\
SE & Cloudiness & 1.56 & 0.6 & exp. \\
& Spatial & 0.25 & 100 & exp. \\
\hline
\end{tabular}

The optimal parameters for the UASIBS and SE models using both cloudiness and spatial covariances computed only using the training data are presented in Table 1. We note that these parameters are optimal for Tucson, AZ. Other areas, sensors, or study periods may require a different parameterization of the error covariances.

In general, the minimum MSE is sensitive to the parameter choice with the most sensitivity shown for $l$ and least sensitivity for $k$. A small change in $l$ ( 0.1 for cloudiness and $10 \mathrm{~km}$ for spatial) typically degrades the MSE by $10 \%$ or more. One exception is the combination of the SE model and spatial covariance which produce MSE surfaces that are less sensitive to a range of parameters, for example a 40 $\mathrm{km}$ difference in $l$ only raises the MSE by $10 \%$.

Large $d$ values for the UASIBS model indicate that the estimated variance from only the clear days is too low. This is because UASIBS suppresses many clouds or slight variations on clear days. An example of this on a cloudy day is shown in Fig. 2 ,

${ }_{410}$ A value of $d<1$ for the SE model indicates that the model tends to overestimate the variance on cloudy days as a result of the tendency to overproduce clouds even at times that should be clear.

Our proposed tuning process is computationally intensive but manageable; computation for one set of $(k, l, d)$ and one cross-validation set using 24 cores of two Intel Xeon E5-2690 v3 processors takes nearly ten minutes. Thus, to tune over the six cross-validation sets, three choices of $k$, ten choices of both $l$ and $d$, spatial and cloudiness correlation parameterizations, and the SE and UASIBS models would take nearly 7 weeks on a single 24 core machine. It would take a typical 4 core laptop or desktop nearly a year to perform the same tuning. To speed up this tuning, the bulk of the operations were converted to GPU code which decreased the run-time for a single parameter set over the test data to five minutes using a single GPU. We used the University of Arizona's El Gato supercomputer, which has 140 NVIDIA K20x GPUs, to perform the tuning in a matter of days. Once tuning is complete, OI can be computed in under five seconds for each image.

\section{Results and Discussion}

435

We compute the OI analysis on each of the images in the verification data set using optimal parameters found in Section 6. First, we compute the analysis of the verification data by only withholding the NREL MIDC GHI sensor at the University 

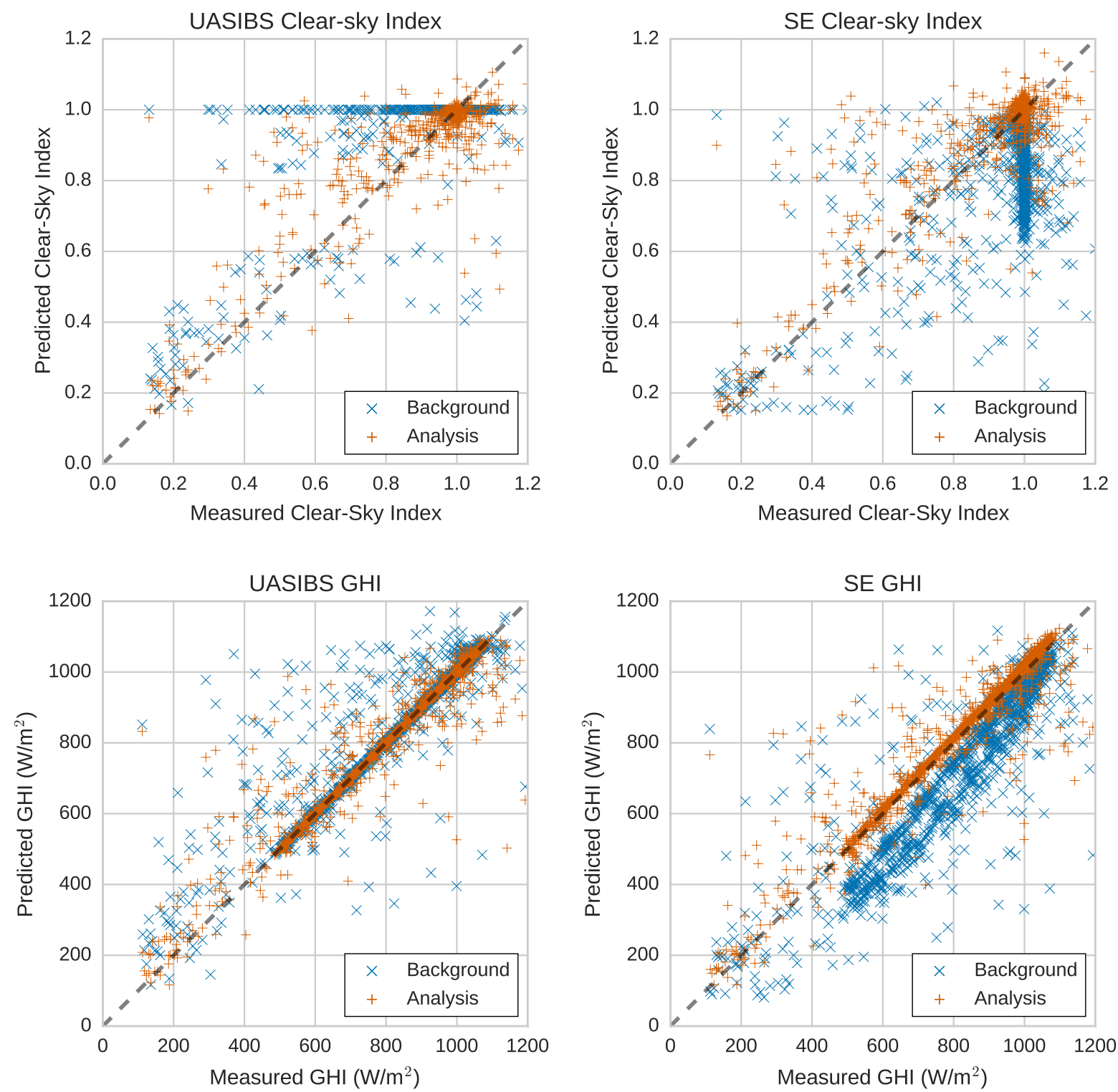

Figure 4: Scatter plots of predicted versus measured clear-sky index (top row) and GHI (bottom row) for the calibrated NREL MIDC GHI sensor on the University of Arizona campus for both the UASIBS model (left column) and SE model (right column). The analysis was computed using cloudiness covariance, the optimal parameters listed in Table 1 and the verification data set. Data from the background images is plotted as blue X's and data from the OI analysis is plotted as orange +'s. GHI is computed by multiplying clear-sky indices and an appropriate clear-sky profile. In each case, we see that the analysis values are more tightly scattered around the dashed $y=x$ line. Also notice that the UASIBS model does not predict clear-sky index values from roughly 0.6 to 0.8 but that the analysis does move some values into this range. 
440 of Arizona, and later we calculate errors while performing six fold cross-validation over the sensors.

Scatter plots of predicted versus measured values at the NREL MIDC sensor using cloudiness covariance are shown in Fig. 4. In the clear-sky index 445 scatter plots, we see that the UASIBS model underpredicts clouds while the SE model over-predicts clouds. It is interesting to note that the UASIBS model does not predict clear-sky index values between 0.6 and 0.8 , and that OI helps to fill in this ${ }_{450}$ gap. The GHI scatter plots show that the analysis performs well and is more tightly scattered around the $y=x$ line with minimal bias. It is especially striking to note how well OI improves the GHI estimates for the SE model. Figures 2 and 4 also 455 demonstrate that OI is not simply a bias correction applied to the whole background because the analysis is not a linear (or even polynomial) function applied to the background values. This is especially evident in the scatter plot of GHI for the SE model 460 (lower right of Fig. 4).

We compute the mean bias error (MBE), mean absolute error (MAE), and root mean squared error (RMSE) over the verification data with five minute average sensor data and "instantaneous" satellite 465 estimates. For RMSE, the square root is computed after all averaging computations. The errors in GHI when only the NREL MIDC sensor was withheld from the OI routine and converting clear-sky index to GHI using the sensor's clear-sky profile are shown 480 470 in Table 2 ,

Table 2: Error statistics for the NREL MIDC sensor on the University of Arizona campus. The analysis was computed with only the MIDC sensor withheld and averaged over the 485 verification data set, and cloudiness covariance was used. Both the UASIBS and SE models show improvements and have a similar analysis RMSE. Units are $\mathrm{W} / \mathrm{m}^{2}$.

\begin{tabular}{lrrr}
\hline & MBE & MAE & RMSE \\
\hline UASIBS Analysis & 4.16 & 27.2 & 71.1 \\
UASIBS Background & 20.7 & 38.8 & 98.8 \\
SE Analysis & 11.2 & 36.0 & 72.7 \\
SE Background & -86.1 & 107 & 140 \\
\hline
\end{tabular}

To calculate the errors over the cross-validation sensors in order to validate OI at locations not included in the algorithm, we averaged over the withheld sensors, the cross-validation runs, and the verification images. Figure 5 shows the reduction in 500 errors for the UASIBS and SE models using cloudiness covariance for the analysis errors as compared

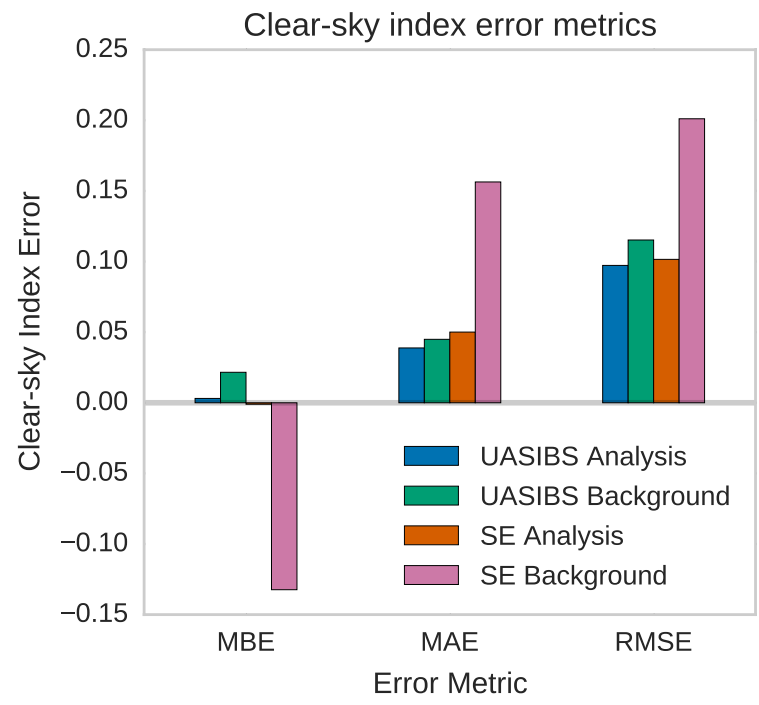

Figure 5: Clear-sky index cross-validation error statistics for the UASIBS and SE models before (background) and after (analysis) performing OI using cloudiness covariance with the optimal parameters listed in Table 1. The error statistics were computed by averaging over the withheld sensors, the cross-validation runs, and the verification times. The SE model initially has a large bias that is corrected by the analysis. After analysis, both the UASIBS and SE models have similar RMSE.

to the background errors. Table 3 presents the errors for the background and analysis computed with each covariance method for the UASIBS and SE models, respectively. Analyzing Figs. 4 and 5 and Tables 2 and 3 , we see that the SE model initially has a large bias that is corrected in the analysis. This also leads to large MAE and RMSE relative improvements of $68 \%$ and $50 \%$, respectively. The analysis using the best covariance parameterization for UASIBS had a RMSE relative improvement of $16 \%$.

Furthermore, it is interesting to see that the errors after optimal interpolation are similar for both the UASIBS and SE models. We interpret this as evidence that one can use the relatively simple semi-empirical model with optimal interpolation and still obtain irradiance estimates that are comparable in quality to estimates from more complicated, physics-based models. This also suggests that the optimal interpolation routine that we have presented is likely to work with satellite image to irradiance models that were not studied here.

OI assumes that the background error is unbiased and Gaussian as described in Eq. (4). However, it is clear from Fig. 5 that the SE background is bi- 
Table 3: Clear-sky index error statistics using the UASIBS and SE models with cloudiness, spatial, or empirical covariance parameterizations. Errors are calculated by averaging over the withheld sensors, cross-validation runs, and verification images. OI using any of the covariance methods improves upon the background for both models. All errors are in units of clear-sky index.

\begin{tabular}{lccr}
\hline UASIBS Model & MBE & MAE & RMSE \\
\hline Cloudiness Analysis & 0.003 & 0.039 & 0.097 \\
Spatial Analysis & 0.004 & 0.038 & 0.099 \\
Empirical Analysis & 0.000 & 0.043 & 0.105 \\
Background & 0.022 & 0.045 & 0.115 \\
& & & \\
SE Model & $\mathrm{MBE}$ & $\mathrm{MAE}$ & $\mathrm{RMSE}$ \\
\hline Cloudiness Analysis & -0.001 & 0.050 & 0.102 \\
Spatial Analysis & -0.005 & 0.051 & 0.105 \\
Empirical Analysis & -0.001 & 0.051 & 0.106 \\
Background & -0.132 & 0.156 & 0.201 \\
\hline
\end{tabular}

ased. From Fig. 4, it also appears that the UASIBS background is not Gaussian. Thus, we cannot as505 sume that this application of OI yielded the best ${ }_{55}$ linear unbiased estimate, but we show that OI still produces measurable improvements.

The results in Table 3 indicate that any of the three methods to compute the background error covariance matrix produce an analysis that improves 560 upon the background. However, when we subjectively compare the analysis of the covariance models, as in Fig. 6, we see that analysis using the cloudiness covariance method better represents the cloud pattern depicted in the visible satellite im- 565 age. Clouds produced using the spatial and empirical covariance methods are physically inconsistent with the clouds depicted in the visible albedo image. For example, the lower left corner of the images in 520 Fig. 6 should have no clouds present according to the visible albedo image, but the empirical covariance analysis has clouds present in that region. The 570 analysis produced using spatial covariance shows a thin and smoothly varying "background cloud" 525 that is simply not observed in the visible albedo image. Furthermore, the cloudiness covariance parameterization is calculated for each satellite image 575 individually which likely leads to a better modeling of the spatial heterogeneity of irradiance. Thus, we recommend the cloudiness covariance parameterization as the method of choice, but additional verification sensors evenly distributed throughout 580 the study area may help to better distinguish the parameterizations through objective measures.

\section{8. Conclusions}

We presented an application of optimal interpolation that combines ground irradiance sensor data with a satellite derived estimate of irradiance. We systematically analyzed three methods to choose 540 an error covariance matrix for the satellite derived GHI estimates. This covariance matrix is critical to the success of OI. We observed the best results by assigning covariances based on the differences in cloudiness rather than spatial or empirical covari545 ances. Our implementation of OI was trained and evaluated using three months of data in Tucson, AZ. We tuned the model parameters over one-third of the data, and presented the results of OI over the remaining two-thirds.

The results show that OI improves the entire satellite derived irradiance field with data from only a small number of point locations. Furthermore, the success of OI with different satellite derived irradiance models indicates that OI is likely applicable to satellite derived irradiance models not described in this paper.

In future work, we wish to study if OI is applicable to larger areas than the city scale studied here. If, for example, clouds form because of the same physical forcings, OI using cloudiness covariance may be able to use sensors in Tucson to improve irradiance estimates 100 miles away in Phoenix. Furthermore, OI as described in this paper can be extended to a Kalman filter with the use of a cloud advection model. This allows forecasts to be made that also incorporate previous satellite and ground sensor data instead of relying on a single snapshot in time.

\section{Acknowledgment}

This project was funded in part by Tucson Electric Power and Arizona Public Service. We thank Technicians for Sustainability, a local solar PV installer, for providing data from rooftop PV systems. ATL thanks the University of Arizona Renewable Energy Network for support. WFH thanks the Department of Energy (DOE) Office of Energy Efficiency and Renewable Energy (EERE) Postdoctoral Research Award for support. This material is based upon work performed on the El Gato supercomputer supported by the National Science Foundation under Grant No. 1228509. This material is 

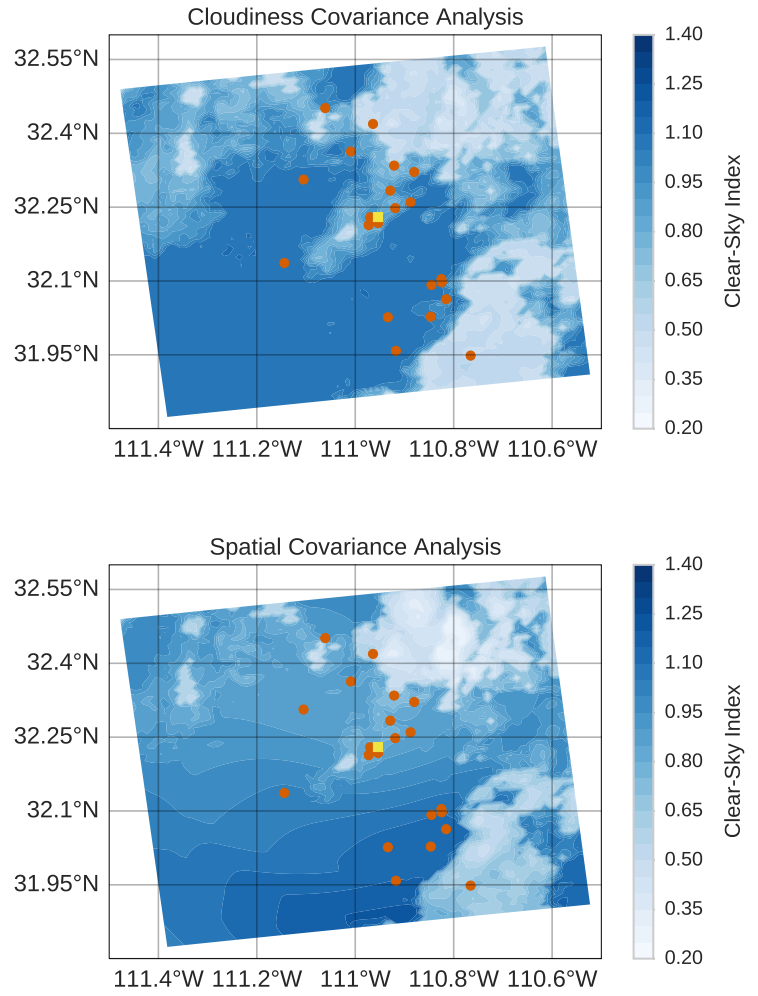

Figure 6: Example of OI using empirical, spatial, and cloudiness covariance with the UASIBS model. The upper left shows the visible image taken from the satellite with the surface albedo removed. The upper right shows the analysis using cloudiness covariance that generally agrees with the visible albedo image. The analysis computed with an empirical covariance matrix (bottom left) generates clouds in the lower left of the image that are not present in the visible albedo image. The spatial covariance analysis (bottom right) shows a smoothly varying and thin "background cloud" that is inconsistent with the visible albedo image.

based upon work supported by the National Science Foundation under Grant No. 1619630.

\section{Supplementary Material}

Location metadata, measurements, clear-sky expectations, and the satellite data used in this study have been released online under the CC0 1.0 license (Lorenzo and Cronin, 2016). The code to generate the optimal interpolation analysis is released under 590 the MIT license (Lorenzo, 2016).

\section{References}

D’Agostino, V., Zelenka, A., 1992. Supplementing solar radiation network data by co-Kriging with satellite images.
International Journal of Climatology 12 (7), 749-761. URL http://dx.doi.org/10.1002/joc.3370120707

Drews, A., de Keizer, A., Beyer, H., Lorenz, E., Betcke, J., van Sark, W., Heydenreich, W., Wiemken, E., Stettler, S., Toggweiler, P., Bofinger, S., Schneider, M., Heilscher, G., Heinemann, D., 2007. Monitoring and remote failure detection of grid-connected PV systems based on satellite observations. Solar Energy 81 (4), 548-564.

口 URL http://dx.doi.org/10.1016/j.solener.2006.06. 019

Escrig, H., Batlles, F., Alonso, J., Baena, F., Bosch, J., Salbidegoitia, I., Burgaleta, J., 2013. Cloud detection, classification and motion estimation using geostationary satellite imagery for cloud cover forecast. Energy 55, 853-859.

URL http://dx.doi.org/10.1016/j.energy.2013.01. 054

Frei, C., Willi, M., Stöckli, R., Dürr, B., 2015. Spatial analysis of sunshine duration in complex terrain by noncontemporaneous combination of station and satellite 
data. International Journal of Climatology 35 (15), 47714790.

URL http://dx.doi.org/10.1002/joc. 4322

Ghonima, M. S., Urquhart, B., Chow, C. W., Shields, J. E., Cazorla, A., Kleissl, J., 2012. A method for cloud detection and opacity classification based on ground based sky imagery. Atmospheric Measurement Techniques 5 (11), 68 2881-2892.

URL http://dx.doi.org/10.5194/amt-5-2881-2012

Journée, M., Müller, R., Bertrand, C., 2012. Solar resource assessment in the Benelux by merging Meteosat-derived climate data and ground measurements. Solar Energy 690 86 (12), 3561-3574.

1] URL http://dx.doi.org/10.1016/j.solener.2012.06. 023

Kalnay, E., 2003. Atmospheric Modeling, Data Assimilation and Predictability. Cambridge University Press.

Kim, C. K., Holmgren, W. F., Stovern, M., Betterton, E. A., 2016. Toward Improved Solar Irradiance Forecasts: Derivation of Downwelling Surface Shortwave Radiation in Arizona from Satellite. Pure and Applied Geophysics. URL http://dx.doi .org/10.1007/s00024-016-1302-3

Kühnert, J., Lorenz, E., Heinemann, D., 2013. SatelliteBased Irradiance and Power Forecasting for the German Energy Market. In: Kleissl, J. (Ed.), Solar Energy Forecasting and Resource Assessment. Elsevier, Ch. 11, $640 \quad$ pp. $267-297$

[1) URL B978-0-12-397177-7.00011-5

Lorenzo, A. T., 2016. alorenzo175/satellite_irradiance_optimal_interpolation: Final research code.

645 URL http://dx.doi.org/10.5281/zenodo.166799 710

Lorenzo, A. T., Cronin, A. D., 2016. Irradiance measurements, satellite images, and clear-sky expectations for Spring 2014 in Tucson, AZ. URL http://dx.doi.org/10.5281/zenodo.166769

650 Lorenzo, A. T., Holmgren, W. F., Cronin, A. D., 2015. Ir- 715 radiance forecasts based on an irradiance monitoring network, cloud motion, and spatial averaging. Solar Energy $122,1158-1169$.

URL http://dx.doi.org/10.1016/j.solener.2015.10. 655 038

Lorenzo, A. T., Holmgren, W. F., Leuthold, M., Kim, C. K., Cronin, A. D., Betterton, E. A., 2014. Short-term PV power forecasts based on a real-time irradiance monitoring network. In: 2014 IEEE 40th Photovoltaic Specialist Conference (PVSC). pp. 0075-0079.

URL http://dx.doi.org/10.1109/PVSC.2014.6925212

Low, K. H., Chen, J., Hoang, T. N., Xu, N., Jaillet, P., 2015. Recent Advances in Scaling Up Gaussian Process Predictive Models for Large Spatiotemporal Data. Springer In665 ternational Publishing, pp. 167-181.

1] URL http://dx.doi.org/10.1007/978-3-319-25138-7_ 16

Mieslinger, T., Ament, F., Chhatbar, K., Meyer, R., 2014. A New Method for Fusion of Measured and Model-derived

670 Solar Radiation Time-series. Energy Procedia 48, 1617- 735 1626 .

URL http://dx.doi.org/10.1016/j.egypro.2014.02. 182

Perez, R., Ineichen, P., Moore, K., Kmiecik, M., Chain, 675 C., George, R., Vignola, F., 2002. A new operational model for satellite-derived irradiances: description and validation. Solar Energy 73 (5), 307-317.

URL http://dx.doi.org/10.1016/S0038-092X(02)
00122-6

680 Polo, J., Martín, L., Vindel, J., 2015. Correcting satellite derived DNI with systematic and seasonal deviations: Application to India. Renewable Energy 80, 238-243.

URL http://dx.doi.org/10.1016/j.renene.2015.02. 031

Polo, J., Wilbert, S., Ruiz-Arias, J., Meyer, R., Gueymard, C., Súri, M., Martín, L., Mieslinger, T., Blanc, P., Grant, I., Boland, J., Ineichen, P., Remund, J., Escobar, R., Troccoli, A., Sengupta, M., Nielsen, K., Renne, D., Geuder, N., Cebecauer, T., 2016. Preliminary survey on site-adaptation techniques for satellite-derived and reanalysis solar radiation datasets. Solar Energy 132, 25-37.

URL http://dx.doi.org/10.1016/j.solener.2016.03. 001

Rasmussen, C., Williams, C., 2005. Covariance Functions. In: Gaussian Processes for Machine Learning. MIT Press, Ch. 4, pp. 79-104.

Reno, M. J., Hansen, C. W., 2016. Identification of periods of clear sky irradiance in time series of GHI measurements. Renewable Energy 90, 520-531.

700 URL http://dx.doi.org/10.1016/j.renene.2015.12. 031

Ruiz-Arias, J. A., Quesada-Ruiz, S., Fernández, E. F., Gueymard, C. A., 2015. Optimal combination of gridded and ground-observed solar radiation data for regional solar resource assessment. Solar Energy 112, 411-424.

URL http://dx.doi.org/10.1016/j.solener.2014.12. 011

Saint-Drenan, Y.-M., Bofinger, S., Rohrig, K., Ernst, B., 2011. Regional Nowcasting of the Solar Power Production with PV-Plant Measurements and Satellite Images. In: Proceedings of the ISES Solar World Congress 2011. International Solar Energy Society, Freiburg, Germany, pp. $1-11$ URL http://dx.doi.org/10.18086/swc. 2011.11.09

Schumann, K., Beyer, H. G., Meyer, R., Chhatbar, K., 2011. Improving Satellite-Derived Solar Resource Analysis with Parallel Ground-Based Measurements. In: Proceedings of the ISES Solar World Congress 2011. International Solar Energy Society, Freiburg, Germany, pp. 1-12. URL http://dx.doi .org/10.18086/swc.2011.24.28

Vicente, G. A., Davenport, J. C., Scofield, R. A., 2010. The role of orographic and parallax corrections on real time high resolution satellite rainfall rate distribution. International Journal of Remote Sensing 23 (2), 221-230. URL http://dx.doi.org/10.1080/01431160010006935

Vignola, F. E., McMahan, A. C., Grover, C. N., 2013. Bankable Solar-Radiation Datasets. In: Kleissl, J. (Ed.), Solar Energy Forecasting and Resource Assessment. Elsevier, Ch. 5, pp. 97-131. URL B978-0-12-397177-7.00005-X

Wilcox, S., Andreas, A., 2010. Solar Resource \& Meteorological Assessment Project (SOLRMAP): Observed Atmospheric and Solar Information System (OASIS); Tucson, Arizona (Data). URL http://dx.doi.org/10.7799/1052226 